\title{
A Single Center Experience for a Feasibility of Totally Laparoscopic Living Donor Right Hepatectomy
}

\author{
Soo Kyung Lee, M.D., Young Seok Han, M.D., Heontak Ha, M.D., Jaryung Han, M.D., Jae Min Chun, M.D. \\ Liver Transplantation and Hepatobiliary Pancreas Surgery, Department of Surgery, Kyungpook National University, School of Medicine, Kyungpook National \\ University Hospital, Daegu, Korea
}

Purpose: Donor safety is the most important problem of living donor liver transplantation (LDLT). Although laparoscopic liver resection has gained popularity with increased surgical experience and the development of laparoscopes and specialized instruments, a totally laparoscopic living donor right hepatectomy (LDRH) technique has not been investigated for efficacy and feasibility. We describe the experiences and outcomes associated with LDRH in adult-to-adult LDLT in order to assess the safety of the totally laparoscopic technique in donors.

Methods: Between May 2016 and July 2017, we performed hepatectomies in 22 living donors using a totally laparoscopic approach. Among them, 20 donors underwent LDRH. We retrospectively reviewed the medical records to ascertain donor safety and the reproducibility of LDRH; intraoperative and post-operative results including complications were demonstrated after performing LDRH.

Results: The median donor age was 29 years old and the median body mass index was $22.6 \mathrm{~kg} / \mathrm{m}^{2}$. The actual graft weight was $710 \mathrm{~g}$ and graft weight/body weight (GRWR) was 1.125. No donors required blood transfusion, conversion to open surgery, or reoperation. The postoperative mortality was nil and postoperative complications were identified in two donors. One had fluid collection in the supra-pubic incision site for graft retrieval and the second had a minor bile leakage from the cutting edge of the right hepatic duct stump. All the liver function tests returned to normal ranges within one month.

Conclusion: LDRH is a feasible operation owing to low blood loss and few complications. However, LDRH can be initially attempted after attaining sufficient experience in laparoscopic hepatectomy and LDLT techniques.

Keywords: Laparoscopic right hepatectomy, Living liver donor, Living donor liver transplantation
Received August 31, 2018

Revised 1st September 27, 2018

2nd October 3, 2018

Accepted October 5, 2018

Corresponding author

Young Seok Han

Liver Transplantation and

Hepatobiliary Pancreas Surgery,

Department of Surgery, Kyungpook

National University, School of

Medicine, Kyungpook National

University Hospital, 130 Dongdeok-

ro, Jung-gu, Daegu 41944, Korea

Tel: $+82-53-200-6734$

Fax: +82-53-421-0510

E-mail: gshys@knu.ac.kr

ORCID:

https://orcid.org/0000-0003-4075-730X

This is an Open Access article distributed under the terms of the Creative Commons Attribution Non-Commercial License (http:/l creativecommons.org/licenses/by-nc/4.0/) which permits unrestricted non-commercial use, distribution, and reproduction in any

Copyright $\odot 2019$ The Journal of Minimally medium, provided the original work is properly cited.

Invasive Surgery. All rights reserved.

\section{INTRODUCTION}

Living donor liver transplantation (LDLT) accounts for about $67 \%$ of total liver transplantation in South Korea because of the lack of organ donations.

However, the huge abdominal wall scar, postoperative pain, long hospital stays, and a long recovery period, have caused hesitation among potential donors, especially young donors. ${ }^{2}$ Thus, pure laparoscopic donor hepatectomy has been suggested as a useful alternative to address the donor's cosmetic problem and fears. ${ }^{3}$

Donor safety is the most important problem in LDLT. The 
major postoperative morbidity rate is estimated to be $9.3 \%$, even in open donor hepatectomy. ${ }^{4}$ Laparoscopic liver resections (LLR) for tumors have gained popularity with the accumulation of experience, better visualization of the operative field with high-quality imaging laparoscopes, and the use of specialized laparoscopic instruments for transecting the liver parenchyma. ${ }^{5,6}$ However, there is a lack of relevant studies investigating the efficacy and feasibility for totally laparoscopic donor right hepatectomy (LDRH). In the Second International Consensus Conference on Laparoscopic Liver Resection, laparoscopic donor major hepatectomy for adult LDLT was classified as IDEAL 2a (development phase) and was not recommended for wide introduction.

Herein, we describe the experiences and outcomes of LDRH in adult-to-adult LDLT to evaluate the safety of the totally laparoscopic technique in donors.

\section{MATERIALS AND METHODS}

The goal of this study was to demonstrate the feasibility of LDRH based on donor safety and reproducibility with standardization of the surgical technique. To ascertain the donor safety and the reproducibility of LDRH, intra-operative and post-operative results including complications were demonstrated after performing LDRH with the same surgical technique by a single surgeon. From January 2015 to July 2017, 128 liver transplantations were performed at Kyungpook National University Hospital. Among these, 79 patients underwent LDLT. From May 2016 to July 2017, 22 donors underwent living donor hepatectomy using a totally laparoscopic approach (Fig. 1), of whom 20 donors underwent LDRH and two donors underwent totally laparoscopic extended left hepatectomy.

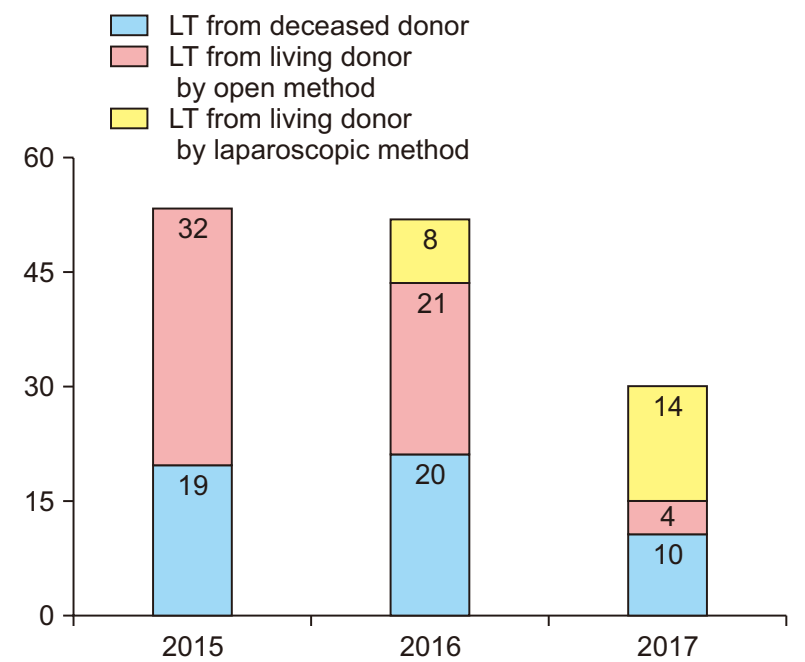

Fig. 1. Annual proportion of donors receiving living donor right hepatectomy (LDRH).
Twenty donors who underwent LDRH were compared with 20 donors who underwent conventional open donor right hepatectomy (CDRH) performed between October 2015 and April 2017. We retrospectively obtained basic information regarding donor hepatectomy, including pre-, intra-, and post-operative data. This study was approved by the institutional Review Board of Kyungpook National University Hospital.

\section{Donor selection process}

LDRH was firstly considered when the recipient's condition was acceptable and the elective LDLT was planned. All donors underwent a pre-operative evaluation, including ultrasonography, dynamic computed tomography (CT) of the liver, and magnetic resonance cholangiopancreatography (MRCP).

And, the donor's hepatic vascular anatomy was a second consideration. Donors with a single portal vein and a single hepatic artery were the primary choice for LDRH, except the 6th donor with 2 hepatic arteries on pre-operative imaging studies. In donor with 2 hepatic arteries, the right posterior hepatic artery of the graft was firstly reconstructed with the recipient's right hepatic artery and the right anterior hepatic artery of the graft was clipped after confirming the good pulsatile back flow. The variations of the hepatic duct were not considered as selection criteria. In addition, we did not include the estimated graft volume of the liver graft as a selection criteria despite the longer operation time, more careful mobilization and dissection of the right liver, and a long skin incision.

Finally, we informed about the benefits and operative risk of this innovative surgery to potential donors who met the above criteria since May 2016, and LDRH was performed only if the donor and family agreed.

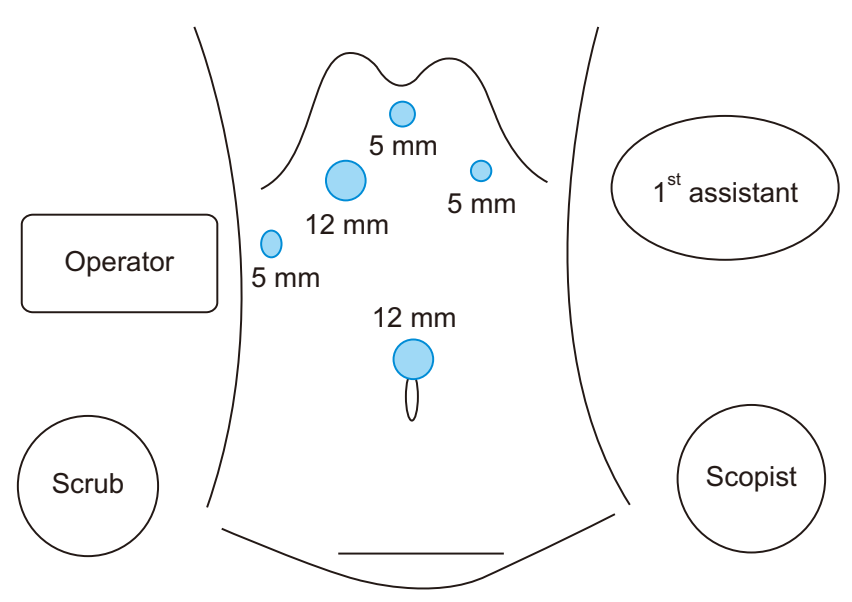

Fig. 2. Trocar placement for a totally laparoscopic living donor right hepatectomy. 


\section{Operation procedure}

For the right hepatectomy procedure, the donors were placed in a left semi-lateral decubitus position with a reversed Trendelenburg position. A 12-mm trocar was inserted for introducing a laparoscope and creating a pneumoperitoneum. In addition, as shown in Fig. 2, a 12-mm trocar, seen in the surgeon's right hand, and three 5-mm trocars were inserted along both subcostal lines. In the first two donors, a 30-degree rigid scope was used, and the remaining 18 donors underwent right hepatectomy via 3-dimensional laparoscopy (Olympus, Tokyo, Japan). The surgeon stood at the right side of the donor. After full mobilization of the liver from the attached ligament with electrocautery hooks or an energy device, the retrohepatic inferior vena cava (IVC) was meticulously dissected (Fig. 3A) and the small sized short hepatic veins were ligated using the
Hem-o-lok (Weck Closure System, Research Triangle Park, NC) or metal clips. After cholecystectomy, the hepatic hilum was exposed and dissected carefully, the right hepatic artery and portal vein were isolated (Fig. 3B, C), and vessel loops were placed around them. The right hepatic artery and right portal vein were temporarily clamped with bull-dog clamps to delineate the parenchyma transection plane along the demarcated line. Liver parenchymal transection was performed using energy devices and a laparoscopic ultrasonic aspirator (CUSA Excel; Valleylab Corp., Boulder, CO). However, the hanging maneuver and the Pringle's maneuver were not used at all during the liver parenchymal transection. Sizable branches of segment 5 and segment 8 of the middle hepatic vein $(\mathrm{MHV})$ were preserved for reconstruction (Fig. 3D, E). After exposure of the hepatic hilar plate (Fig. 3F), intraoperative cholangiography (IOC) was performed for the pre-
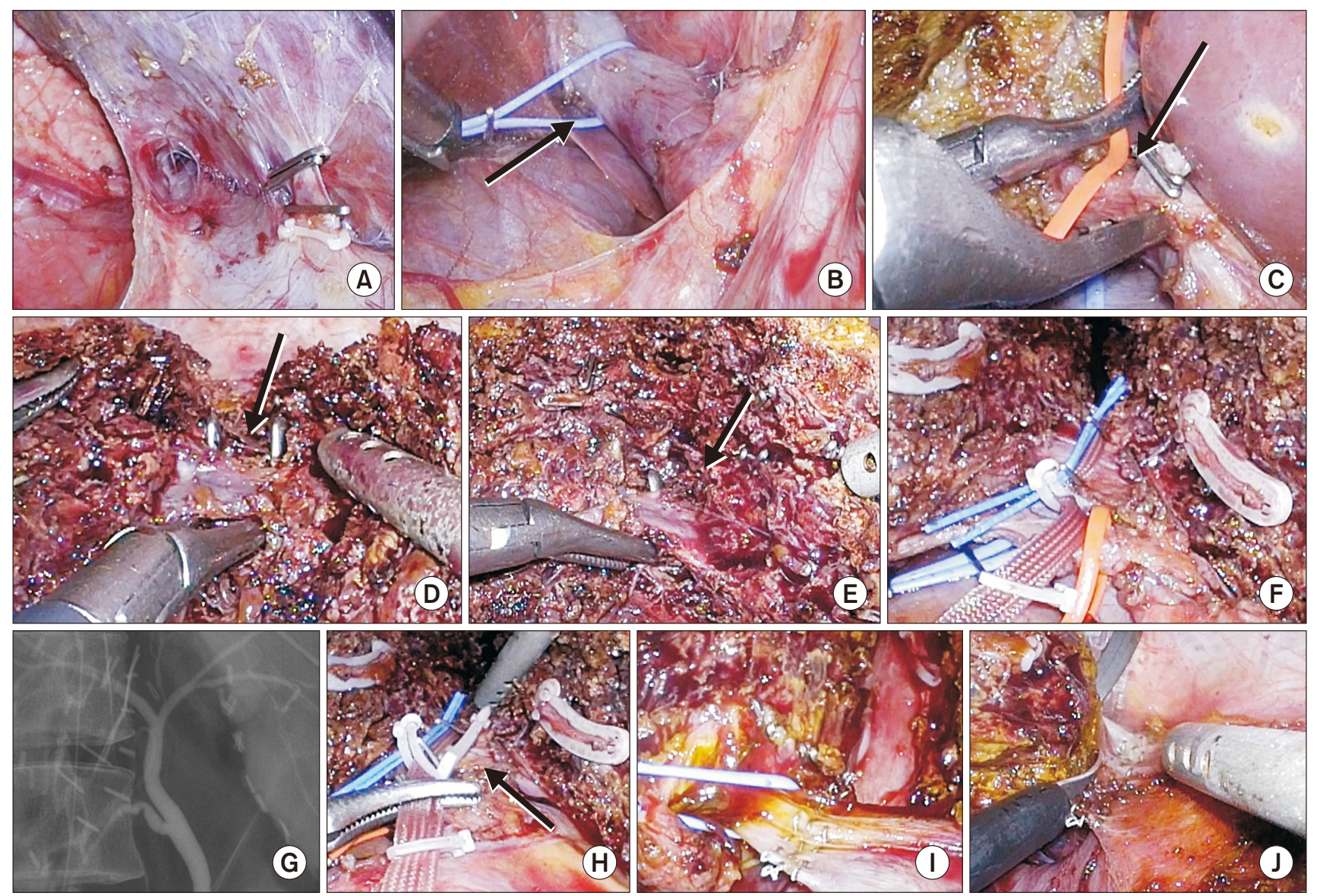

Fig. 3. Operative procedure. After mobilizing right hemiliver, the retrohepatic inferior vena cava (IVC) was meticulously dissected and the short hepatic vein was ligated with Hem-0-lok or metal clips $(A)$. Right portal vein $(B)$ and hepatic artery $(C)$ were encircled with vessel loops. During liver parenchymal transection, segment 8 and 5 branches (D, E) from the middle hepatic vein (MHV) was isolated for the reconstruction on the back table. After the right hepatic hilar plate was exposed, a radiopaque rubber marker band was affixed at an adequate point (F). The cutting line of right hepatic duct was confirmed (G) under intraoperative cholangiography (IOC) guidance and clipped with Hem-o-lok clip (H). The right portal vein (I) and hepatic vein (J) were transected with the stapling. 
cise cutting of hepatic duct and the confirmation of safety of donor's remnant hepatic ducts (Fig. 3G). To verify the optimal right hepatic duct division point, a radiopaque rubber marker band was affixed at an adequate point and the 1st IOC was performed. The right hepatic duct was clamped with a Hemoo-lok clip and was cut just before the clip after determining the optimal clamping and confirming the cutting line under the 2nd IOC (Fig. 3H). In nine donors, two right hepatic ducts were identified on pre-operative MRCP. The two hepatic ducts were divided using a procedure similar to that described in our previous report. ${ }^{8}$ After completion of parenchymal transection, a 10 12 cm horizontal skin incision was made just above the symphysis pubis (Pfannenstiel incision), without opening the peritoneum, for retrieval of the graft. $12 \mathrm{~mm}$ tro-

Table 1. The demographic characteristics of LDRH group, and comparative results between CDRH and LDRH group

\begin{tabular}{|c|c|c|c|}
\hline & $\operatorname{CDRH}(n=20)$ & LDRH $(n=20)$ & $p$ value \\
\hline Age, mean $\pm S D$, years & $36.1 \pm 14.6$ & $32.4 \pm 12.1$ & 0.391 \\
\hline Sex ratio (M:F) & $12: 8$ & $13: 7$ & 0.744 \\
\hline$B M I$, mean $\pm S D, k g / m^{2}$ & $23.7 \pm 3.8$ & $23.3 \pm 3.0$ & 0.737 \\
\hline \multicolumn{4}{|l|}{ Relationship, n } \\
\hline Son/Daughter & $9 / 2$ & $9 / 2$ & \\
\hline Father & 0 & 1 & \\
\hline Husband/Wife & $0 / 3$ & $2 / 1$ & \\
\hline Brother/Sister & $3 / 2$ & $0 / 2$ & \\
\hline Others (Nephew, Daugher-in-law) & 1 & 3 & \\
\hline \multicolumn{4}{|l|}{ Intraoperative results } \\
\hline Estimated graft weight, mean $\pm S D, g$ & $733 \pm 136.7$ & $758.0 \pm 139.9$ & 0.574 \\
\hline Actual graft weight, mean $\pm S D, g$ & $690.5 \pm 133.6$ & $721.0 \pm 132.3$ & 0.473 \\
\hline Estimated remnant liver volume, mean $\pm S D, \%$ & $37.9 \pm 4.4$ & $34.7 \pm 2.8$ & 0.011 \\
\hline Estimated GRWR, mean $\pm S D$ & $1.0 \pm 0.1$ & $1.1 \pm 0.2$ & 0.017 \\
\hline Acutal GRWR, mean $\pm S D$ & $0.95 \pm 0.18$ & $1.13 \pm 0.27$ & 0.019 \\
\hline Total operation time, mean $\pm S D$, min & $319.6 \pm 70.1$ & $391.4 \pm 76.3$ & 0.004 \\
\hline Warm ischmic time ${ }^{*}$, mean $\pm S D$, min & $1.4 \pm 0.5$ & $7.4 \pm 3.1$ & $<0.001$ \\
\hline Intraoperative RBC transfusion, n $(\%)$ & $1(5)$ & 0 & 0.235 \\
\hline Open Conversion, $n$ & & 0 & \\
\hline \multicolumn{4}{|l|}{ Postoperative outcomes } \\
\hline Peak AST, mean $\pm S D$, IU/L & $157.8 \pm 99.2$ & $193.1 \pm 75.6$ & 0.213 \\
\hline Peak ALT, mean $\pm S D$, IU/L & $141.4 \pm 84.0$ & $223.0 \pm 97.2$ & 0.007 \\
\hline Peak Total bilirubin, mean $\pm S D$, mg/L & $2.83 \pm 1.0$ & $2.89 \pm 1.1$ & 0.873 \\
\hline Peak Prothrombin time, mean \pm SD, INR & $1.40 \pm 0.12$ & $1.40 \pm 0.12$ & 0.950 \\
\hline \multicolumn{4}{|l|}{ Postoperative complications ${ }^{\dagger}, \mathrm{n}$} \\
\hline Grade I & 0 & 1 & \\
\hline Grade Illa & 0 & 1 & \\
\hline Hospital stay, mean $\pm S D$, days & $8.25 \pm 1.4$ & $8.75 \pm 2.0$ & 0.382 \\
\hline
\end{tabular}

$\mathrm{LDRH}=$ laparoscopic donor right hepatectomy; $\mathrm{CDRH}=$ conventional open donor right hepatectomy; $\mathrm{SD}=$ standard deviation; $\mathrm{BMI}=$ body mass index; GRWR = graft weight/body weight of recipient ${ }^{*} 10 ; \mathrm{RBC}=$ red blood cell; AST = aspartate aminotransferase; ALT = alanine aminotransferase; INR = international normalized ratio. ${ }^{*}$ Warm ischemic time was considered as time from the stapling of right portal vein to the perfusion of HTK solution. ${ }^{\dagger}$ Complications were graded according to the classification system proposed by Clavian. ${ }^{9}$ 
car was placed through this supra-pubic incision to insert a large sized bag. The transected right liver was put in the bag for easy retrieval and to decrease the ischemic time. The right hepatic artery was ligated with two Hem-o-lok clips and the right portal vein was cut with unilateral linear staplers (endoTA; Covidien, Dublin, Ireland). The large-sized ( $<5 \mathrm{~mm})$ right inferior hepatic veins (RIHV) and the right hepatic veins (RHV) were also transected with unilateral linear staplers for reconstruction (Fig. 3I, J). The graft was retrieved through the supra-pubic incision and flushed on the back table with $2 \mathrm{~L}$ of histidine-tryptophan-ketoglutarate (HTK) solution, which was mixed with $2000 \mathrm{U}$ of heparin. The supra-pubic incision was closed, and $\mathrm{CO}_{2}$ gas was re-insufflated to check for hemostasis and biliostasis. After anchoring the falciform ligament, a closed suction drain was inserted. A follow-up CT scan was routinely performed on postoperative day 6 and the donors were discharged on postoperative day 7. The donors were followed up in about 6 months to check the liver function tests and for a follow-up CT scan.

\section{Statistical analysis}

All results were expressed as mean \pm standard deviation. Continuous variables were compared using Student $t$ tests, and categorical variables were compared using the chi-square test or Fisher exact test. A 2-tailed $p<.05$ was considered statistically significant. All statistical analyses were performed using SPSS software (version 22; SPSS Inc., Chicago, IL)

\section{RESULTS}

\section{Demographics}

The detailed characteristics of LDRH donors are summarized in Table 1. The mean age was 32.4 (17 64) years and 13 were male. The mean body mass index was 23.3 (19.6 31.8) $\mathrm{kg} / \mathrm{m}^{2}$. The estimated graft weight was $734(595 \sim 1,077) \mathrm{g}$ and the estimated remnant liver volume of donors was 34.7 (30 40.8) \%. The actual graft weight was $721(510 \sim 920) \mathrm{g}$. The estimated graft weight/body weight (GRWR) and actual GRWR were $1.1(0.68 \sim 1.69)$ and $1.13(0.79 \sim 1.63)$, respectively.

\section{Intraoperative data}

None of these donors required blood transfusion, conversion to open surgery, or reoperation. The operation time from skin incision to skin closure was 391 (223 530) minutes. The average time from clamping of the right portal vein to reperfusion of HTK solution was 7.4 (2 13) minutes.

Table 2 demonstrates hepatic anatomical variations in do-
Table 2. Hepatic anatomical variations in donors

\begin{tabular}{|c|c|}
\hline & Number $(\%)$ \\
\hline \multicolumn{2}{|l|}{ Right hepatic artery } \\
\hline Single artery & 19 \\
\hline Two arteries* & 1 \\
\hline \multicolumn{2}{|l|}{ Right portal vein } \\
\hline Single vein & 20 \\
\hline \multicolumn{2}{|l|}{ Hepatic veins } \\
\hline V5 of more than $5 \mathrm{~mm}$ & 16 \\
\hline V8 of more than $5 \mathrm{~mm}$ & 14 \\
\hline Right inferior hepatic vein & 7 \\
\hline \multicolumn{2}{|l|}{ Right hepatic duct } \\
\hline Normal anatomy & $12(60 \%)$ \\
\hline Trifurcated biliary anatomy & $3(15 \%)$ \\
\hline $\begin{array}{l}\text { Right anterior hepatic duct opening into } \\
\text { common hepatic duct }\end{array}$ & $2(10 \%)$ \\
\hline $\begin{array}{l}\text { Right posterior hepatic duct opening into left } \\
\text { hepatic duct }\end{array}$ & $3(15 \%)$ \\
\hline
\end{tabular}

* Right posterior hepatic artery was originating from the superior mesenteric artery.

nors. V5 or V8 of more than $5 \mathrm{~mm}$ for venous drainage of right anterior section were successfully reconstructed with artificial graft in 19 donors. The reconstructed MHV and RHV were anastomosed with the recipient's RHV orifice after oneorifice venoplasty. The RIHV requiring reconstruction were identified in nine donors and were successfully anastomosed with the recipient's inferior vena cava without venoplasty.

The right portal vein was conventionally reconstructed using the recipient's main portal vein. The short segment of the right portal vein was identified in one donor; it was well reconstructed without special venoplasty.

One graft with two hepatic arteries was reconstructed by using the right and left hepatic artery of the recipient. The eight grafts of all included donors had right hepatic ducts with two orifices which were reconstructed using the common hepatic duct of the recipients after ductoplasty.

\section{Postoperative results}

The postoperative mortality was nil. Postoperative complications were identified in two donors. One complication was fluid collection in the supra-pubic incision site for graft retrieval. This fluid collection was simply resolved by a single needle aspiration. The other complication was a minor bile 

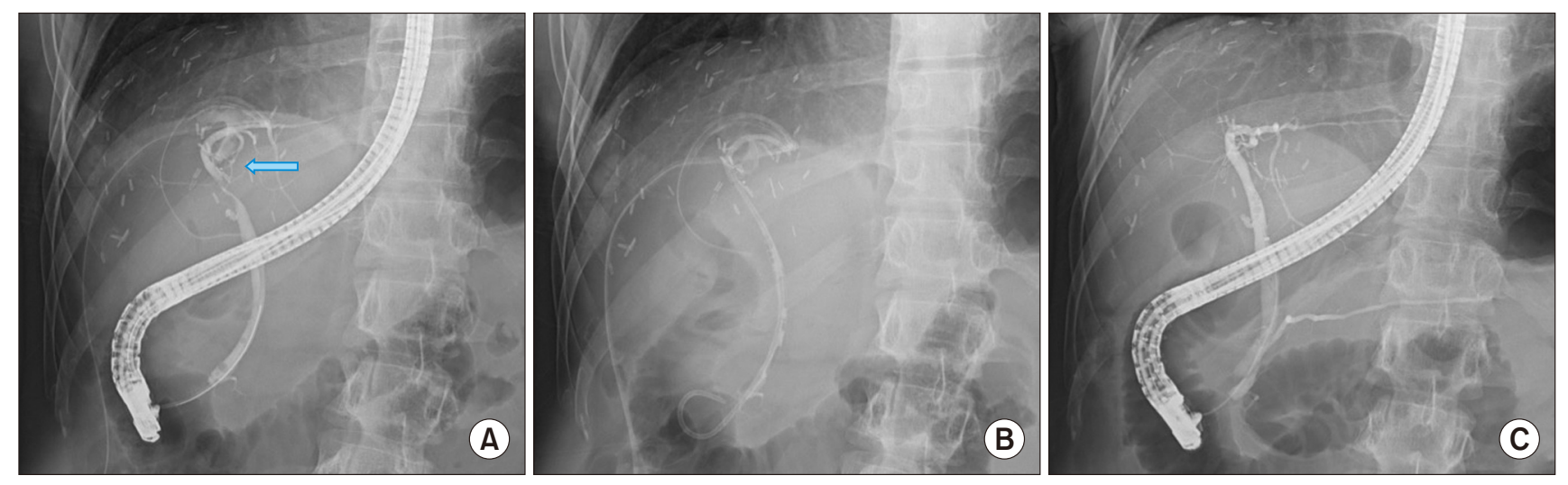

Fig. 4. Bile leakage from the cutting edge of the right hepatic duct stump was identified by endoscopic retrograde cholangiography (A) and a biliary stent was inserted (B). After 3 months, the bile leakage was completely resolved (C).

leakage from the cutting edge of the right hepatic duct stump which was cured by endoscopic biliary stenting (Clavien IIIa) (Fig. 4). ${ }^{9}$ The mean peak AST levels and serum total bilirubin levels were 193 (87 394) IU/L and 2.89 (1.54 5.23) mg/ $\mathrm{dl}$, respectively, during the immediate postoperative period. All liver function tests returned to normal ranges within one month. Liver regeneration was confirmed by CT or ultrasonography in all donors at about six months after donation. The mean hospital length of stay was $8.75(6 \sim 15)$ days.

\section{LDRH vs CDRH}

Table 1 summarizes the demographic characteristics and postoperative outcomes of both CDRH and LDRH. The estimated remnant liver volume was statistically larger in the CDRH group (37.9\% vs 34.7\%; $p=.011$ ), but GRWR was higher in the LDRH group (1.0 vs $1.1 ; p=.017)$. The total operation time (319.6 vs 391.4 minutes; $p=.004$ ), and warm ischemic time from the stapling of right portal vein to the perfusion of HTK solution (1.4 vs 7.4 minutes; $p<.001$ ) was significantly longer in the LDRH than in the CDRH. During early postoperative period, peak alanine aminotransferase (ALT) was significantly higher in the LDRH group (141 IU/L vs $223 \mathrm{IU} / \mathrm{L} ; p=.007$ ), but there was no statistical difference in aspartate aminotransferase (AST) and total bilirubin.

One case of the CDRH group received a single unit red blood cell (RBC) transfusion due to intraoperative hemorrhage, but none of the donors in the LDRH group required a transfusion during surgery or postoperative period. The postoperative complication was identified in 2 cases of the LDRH group, but there was no complication in the $\mathrm{CDRH}$ group. One donor with bile leakage in the LDRH group discharged at 15 days after hepatectomy, but there was no statistical difference in postoperative hospital stay between both groups.

\section{DISCUSSION}

The scarcity of donor organs is the limiting factor in liver transplantation. After the introduction of LDLT for adult patients with end-stage liver disease, donor hepatectomy from a healthy person has been performed as one of the means to expand organ availability. However, donors are at risk of significant morbidity following hepatectomy. Furthermore, while mortality rates among donors are low, early death and acute liver failure can occur.

Donor safety is the most important issue in LDLT despite the surgeon's efforts to improve the donor's quality of life. Therefore, strict criteria should be applied because of the safety of the donor and the potential failure of the liver transplant due to the difficulties of the operation. Early reports of pure laparoscopic donor hepatectomy suggest the following selection criteria for donation of the right liver: single and longer segments in the right hepatic artery; right portal veins and right hepatic duct; donors with fewer segment 5 and 8 branches of the hepatic vein; and no large inferior hepatic veins. ${ }^{3}$

Laparoscopic major hepatectomy showed improved intraoperative and peri-operative results with experience, including decreased operating time, lower blood loss, less use of the Pringle's maneuver, lower conversion rate, and shorter length of hospital stay. ${ }^{10}$ However, experts have stated that laparoscopic donor right hepatectomy is still innovative and in the developmental stage, and can only be recommended to be performed by surgeons experienced in both LLR and LDLT. ${ }^{11}$

In Korea, Han's group announced world's first LDRH in $2015,{ }^{12}$ and Kim et al. ${ }^{3}$ reported the feasibility of LDRH in a short series of 3 cases. Since then, several major liver transplant centers in Korea have started to present short-term results of LDRH based on sufficient experience. ${ }^{13,14}$

Careful preparation and blood-saving surgery will signifi- 
cantly lower the postoperative morbidity. To reduce bleeding during hepatectomy, it is important to decrease the central venous pressure (CVP). Bleeding from the hepatic veins can be minimized by maintaining a low CVP. Reducing the airway pressure is also effective for controlling bleeding from the hepatic vein and is safer than increasing the pneumoperitoneum. $^{15,16}$

We used laparoscopic coagulation shears to divide the superficial layer of the liver. In addition, deeper transection was performed using an ultrasonic surgical aspirator to meticulously expose the intraparenchymal structures. Reduction of blood loss is essential during liver parenchymal transection. Although controversial in laparoscopic hepatectomy, the application of Pringle's maneuver can help reduce blood loss. However, because donor livers were healthy and unexpected hemorrhage was prevented with the help of low CVP and meticulous parenchymal dissection, Pringle's maneuver was not used at all. Actually, in our center, compared with CDRH, there was no blood transfusion and open conversion because of massive bleeding in LDRH, even though there was no statistical significance.

The division of the right hepatic duct is one of the most important steps during donor hepatectomy for not the outcome of the anastomosis in the recipient and the safety of the donor. Although a preoperative MRCP provides reliable information about the anatomy of the biliary tree, a laparoscopic intraoperative cholangiogram should be performed to accurately divide the hepatic ducts and identify whether the donor's remnant hepatic duct is injured. We observed bile leakage from the division site of the right hepatic duct despite using an intraoperative cholangiogram. The clips that are used to close the bile duct or vessels may sometimes be missed, and thereby, bile leakage or bleeding can occur. Leaving a little stump or double clipping may prevent these problems, but the right hepatic duct of the graft can be very short or two orifices can inevitably develop. Cutting and suturing the division site of the bile duct may be safer, along with shifting of the bile duct division line towards the left side. However, this procedure requires more time and a sufficient learning curve for laparoscopic intracorporeal suturing. To overcome these drawbacks, highly experienced donor surgeons with excellent skills in performing laparoscopic hepatectomy are required.

The conventional intraoperative cholangiogram under the radiologic field is an indirect imaging technique. Hence, we were not able to divide and close the hepatic duct under a direct visual field. Recently, various methods for the closure of hepatic duct stump have been attempted to avoid delayed leakage of bile or hepatic duct stenosis, such as the usefulness of indocyanine-green (ICG) fluorescence cholangiography as reported by Hong et al. ${ }^{13}$ By them, ICG near-infrared fluores- cence cholangiography may have played a role in saving time, thereby enabling rapid dissection and increasing the surgeon's confidence in confirming the accurate bile duct division line.

Compared with $\mathrm{CDRH}$, even if there is a statistical difference in the estimated remnant liver volume and GRWR between the two groups, it would not be possible to give meaning because they were within the accepted range for donation. Total operation time and warm ischemic time was significantly longer in LDRH. But, the longer operation time did not affect the postoperative course of the donors. In order to lessen the warm ischemic time, we performed firstly the pre-incision of the skin and fascia to take out the right liver and put the transected right liver in the pocket before the closure of right hepatic inflow. By performing this preparation, we were able to staple and cut liver vessels and perfuse the HTK solution, within 10 minutes, ensuring the safety of the graft.

According to Kim et al. ${ }^{3}$, liver grafts exceeding $650 \mathrm{~g}$ were excluded for LDRH. However, we didn't consider the estimated graft volume as one of donor selection criteria for LDRH based on sufficient experience of laparoscopic major hepatectomy. Actually, if the graft volume is large, careful liver traction and dissection are indispensable because of the high injury risk of liver, major vessels, and diaphragm during the mobilization of the right liver. In addition, it is important to have enough incision length for safe retrieval of the liver graft. Hence, we performed the suprapubic incision up to $12 \mathrm{~cm}$ in proportion to the liver graft size.

In one donor, fluid collection in the supra-pubic incision site was identified. However, the donors' satisfaction with respect to the cosmetic outcomes and reduced postoperative pain were very high and laparoscopic donor hepatectomy seems to have contributed to reduced anxiety about donation in both donors and recipients. Nevertheless, there are obvious limitations for LDRH because of the lack of experience and sufficient data.

In conclusion, LDRH is a feasible operation because of reduced blood loss and few complications. However, the long operating time and the increased risk of bile duct injury due to the limitations of laparoscopic hepatectomy are factors that need to be overcome in the future. In addition, continuous technical development and building experience for standardization of the technique may be warranted in the future.

\section{ORCID}

Young Seok Han, https://orcid.org/0000-0003-4075-730X

Heontak Ha, https://orcid.org/0000-0001-6280-0749

Jaryung Han, https://orcid.org/0000-0003-4812-2659 


\section{CONFLICT OF INTEREST}

None.

\section{FUNDING}

None.

\section{ACKNOWLEDGMENTS}

None.

\section{REFERENCES}

1) Suh KS, Suh SW, Lee JM, Choi Y, Yi NJ, Lee KW. Recent advancements in and views on the donor operation in living donor liver transplantation: a single-center study of 886 patients over 13 years. Liver Transpl 2015;21:329-338.

2) Cherqui D, Soubrane O, Husson E, et al. Laparoscopic living donor hepatectomy for liver transplantation in children. Lancet 2002; 359:392-396.

3) Kim KH, Kang SH, Jung DH, et al. Initial Outcomes of Pure Laparoscopic Living Donor Right Hepatectomy in an Experienced Adult Living Donor Liver Transplant Center. Transplantation 2017;101:1106-1110.

4) Lee JG, Lee KW, Kwon CHD, et al. Donor safety in living donor liver transplantation: The Korean organ transplantation registry study. Liver Transpl 2017;23:999-1006.

5) Han HS, Cho JY, Yoon YS. Techniques for performing laparoscopic liver resection in various hepatic locations. J Hepatobiliary Pancreat Surg 2009;16:427-432.

6) Guro H, Cho JY, Han HS, Yoon YS, Choi Y, Periyasamy M. Current status of laparoscopic liver resection for hepatocellular carci- noma. Clin Mol Hepatol 2016;22:212-218.

7) Wakabayashi G, Cherqui D, Geller DA, et al. Recommendations for laparoscopic liver resection: a report from the second international consensus conference held in Morioka. Ann Surg 2015;261: 619-629.

8) Han YS, Ha H, Kwon HJ, Chun JM. Pure laparoscopic donor right hepatectomy in a living donor with type 3a biliary variation: A case report. Medicine (Baltimore) 2017;96:e8076.

9) Clavien PA, Barkun J, de Oliveira ML, et al. The Clavien-Dindo classification of surgical complications: five-year experience. Ann Surg 2009;250:187-196.

10) Dagher I, O’Rourke N, Geller DA, et al. Laparoscopic major hepatectomy: an evolution in standard of care. Ann Surg 2009;250:856860.

11) Han HS, Cho JY, Kaneko H, et al. Expert Panel Statement on Laparoscopic Living Donor Hepatectomy. Dig Surg 2018;35:284288.

12) Han HS, Cho JY, Yoon YS, et al. Total laparoscopic living donor right hepatectomy. Surg Endosc 2015;29:184.

13) Hong SK, Lee KW, Choi Y, et al. Initial experience with purely laparoscopic living-donor right hepatectomy. Br J Surg 2018;105: 751-759.

14) Kwon CHD, Choi GS, Kim JM, et al. Results of consecutive 54 cases of purely laparoscopic donor hepatectomy for adult living donor liver transplantation recipients. Liver Transpl 2018 Jul 18 [Epub]. DOI: 10.1002/1t.25307.

15) Melendez JA, Arslan V, Fischer ME, et al. Perioperative outcomes of major hepatic resections under low central venous pressure anesthesia: blood loss, blood transfusion, and the risk of postoperative renal dysfunction. J Am Coll Surg 1998;187:620-625.

16) Kobayashi S, Honda G, Kurata M, et al. An Experimental Study on the Relationship Among Airway Pressure, Pneumoperitoneum Pressure, and Central Venous Pressure in Pure Laparoscopic Hepatectomy. Ann Surg 2016;263:1159-1163. 